\title{
ERRATUM
}

\section{Adding nebivolol to ongoing antihypertensive therapy improves blood pressure and response rates in patients with uncontrolled stage I-II hypertension}

JM Neutel, DHG Smith and AH Gradman

Journal of Human Hypertension (2010) 24, 430; doi:10.1038/jhh.2010.34

Correction to: Journal of Human Hypertension (2010) 24, 64-73; doi:10.1038/jhh.2009.33

Owing to an error during typesetting, the acronym AMBP on page 71, column 1, line 12, was incorrectly written and should read ABPM (ambulatory BP monitoring).
The publishers would like to apologize for this mistake. 